\title{
Didaktik des Schreibens - vom Produkt zum Prozess und weiter zur Textkompetenz
}

\section{Peter Sieber}

Wie hat sich die Diskussion um die Didaktik des Schreibens in den letzten Jahrzehnten im deutschsprachigen Raum entwickelt? Wichtige Entwicklungslinien werden mit einem spezifischen Augenmerk auf die Diskussionen und Beiträge aus der Deutschschweiz nachgezeichnet. In der produktiven Kooperation von Schreibforschung und Schreibdidaktik ist eine empirische Fundierung der Schreibdidaktik möglich geworden. Dabei hat der Blick auf den Schreibprozess in den vergangenen Jahren einen zentralen Stellenwert erhalten. Eine "prozessorientierte Schreibdidaktik» entwickelte auf diesen Grundlagen eine Vielzahl von Anregungen für einen erfolgreichen Schreibunterricht. In jüngster Zeit werden - auch im Licht der öffentlichen Thematisierung von schulischen Leistungen - die erzielten Effekte stärker wahrgenommen und diskutiert: Mit der Notwendigkeit einer breiten Literalitätsförderung wird der Aufbau von Textkompetenz zu einem zentralen Untersuchungsgegenstand.

\section{Zur Einführung}

Um die Diskussionen der Schreibdidaktik der letzten Jahrzehnte auszuleuchten, wird eine dreifache Zugriffsweise gewählt, die es möglich machen soll, in einem letzten Teil die aktuellen Entwicklungen zu verorten:

Als erstes werden zentrale Elemente der Auseinandersetzung mit der Deutschdidaktik umrissen. Der (deutschsprachige) fachdidaktische Diskurs zum Schreiben ist eingebettet einerseits in den engeren Zusammenhang der Deutschdidaktik, andererseits in den weiteren Horizont des Spannungsfelds von Fachdidaktik und Fachwissenschaft, Allgemeiner Didaktik und Erziehungswissenschaft sowie deren Bezüge zum schulischen Feld. ${ }^{1}$

Als Zweites werden spezifische Elemente der deutschschweizerischen Diskussion herausgestellt, die aus einer vorgängigen Abstinenz von der internationalen Diskussion schliesslich zur Integration und Mitgestaltung des deutschdidaktischen Diskurses führten. 
Diese Integration lässt sich mit einem kooperativen Zusammengehen von Schreibforschung und Schreibdidaktik beschreiben, die das Konzept einer prozessorientierten Schreibdidaktik entwickelt und in neueren Lehrwerken und Unterrichtsanregungen implementiert hat.

Die Auseinandersetzung mit aktuellen Entwicklungen, wie sie aus den Diskussionen um Literalitätsförderung, Bildungsstandards und Kompetenzmodelle resultieren, bildet den Abschluss der Darstellung: Die Dimensionen der Textualität und der Textkompetenz erhalten neues Gewicht.

\section{Entwicklungen des Diskurses in der Deutsch- didaktik}

Im Jahr 2003 ist ein zweibändiges Handbuch «Didaktik der deutschen Sprache» erschienen (Bredel, Günther, Klotz, Ossner \& Siebert-Ott, 2003). Darin werden - mit dem Anspruch, «den klassischen Stand (...) zunächst einmal als solchen [zu] etablieren» (S. 11) im Bereich des Schreibens insgesamt acht verschiedene Aspekte thematisiert. ${ }^{2}$ Die Breite der Darstellung und die darin verarbeitete Literatur sowie die Fülle der beschriebenen Projekte und Untersuchungen lassen ein Fazit zu, wie es Otto Ludwig am Ende seines Artikels Geschichte der Didaktik des Texteschreibens festhält:

Damit wird die Didaktik des Texteschreibens selber zum Gegenstand der Forschung. Insgesamt kann man also konstatieren, dass die Didaktik des Texteschreibens auf dem Wege ist, auch eine empirische Wissenschaft zu werden. (Ludwig, 2003a, S. 176).

Bis es so weit kommen konnte, war eine intensive Auseinandersetzung mit den Zielen, Vorgehensweisen und Kooperationsmöglichkeiten der Deutschdidaktik nötig, wie sie sich v.a. seit Mitte der 70er-Jahre des letzten Jahrhunderts feststellen lässt.

Die Entwicklung aus der Perspektive der Fachdiskussion Die Deutschdidaktik hat sich besonders in den 70er- und frühen 80er-Jahren intensiv mit Fragen der Konstitution des Faches und der Situierung innerhalb der Scientific Community auseinandergesetzt. Stark vergröbert lässt sich die Entwicklung des deutschdidaktischen Diskurses wie folgt charakterisieren. ${ }^{3}$

Bis gegen Ende der 60er Jahre herrschte das vor, was in der deutschdidaktischen Diskussion als "Tradierung didaktischen Brauchtums» (Becker-Mrotzek, 1997b, S. 17) bezeichnet wird. Ausgangspunkt waren dabei eigene und fremde Erfahrungen mit Deutschunterricht und Lehrerbildung. Praktisches, vor allem methodisches Wissen über Lehr-/Lernverfahren wurde dabei ins Verhältnis gesetzt zum theoretischen Wissen über Sprache und Literatur, wie es in den Fachwissenschaften erarbeitet und diskutiert wurde. Voraussetzung für fachdidaktisches Arbeiten war aber in erster Linie erfolgreiche Unterrichtserfahrung und 
weniger die Kenntnis relevanter sprachwissenschaftlicher und didaktischer Zusammenhänge.

Das Verhältnis von Sprachwissenschaft und Sprachdidaktik zu jener Zeit wird in der Fachgeschichte mit einem Zwei-Phasen-Schema (Eichler \& Henze, 1990, S. 153) charakterisiert: Die Fachwissenschaft erarbeitet die Inhalte, sie kümmert sich darum, was gelehrt werden soll; die Fachdidaktik, verstanden vorwiegend als Methodik, als Lehre von der Umsetzung vorgegebener Inhalte in Unterrichtsstoffe, arbeitet die vorgegebenen Inhalte für die entsprechenden Schulstufen auf, sie beschäftigt sich also vor allem damit, wie gelehrt werden soll. - In dieser Zeit war damit der Einfluss der Sprachwissenschaft auf Sprachdidaktik und Deutschunterricht ebenso stark wie unreflektiert.

Mit dem Ausbau von Sprachwissenschaft und Sprachdidaktik an den deutschen Hochschulen zu Beginn der 70er-Jahre veränderte sich dies: Neuere sprachwissenschaftliche Theorien wurden von der Sprachdidaktik aus befragt auf Umsetzungsmöglichkeiten für die Schule und - manchmal nur allzu vorschnell - in Lehrmittel und Unterrichtsanregungen umgegossen. So ist es z. B. frühen Ansätzen der Transformationsgrammatik ergangen, so lässt sich auch die dominierende Stellung des Rollenspiels in der Didaktik des mündlichen Unterrichts in der zweiten Hälfte der Siebzigerjahre erklären.

Die zunehmende Etablierung der Fachdidaktik in den 70er-Jahren führte allmählich zu einem differenzierteren Verhältnis von Sprachwissenschaft und Sprachdidaktik. Nicht mehr allein die Umsetzung von fachwissenschaftlichem Wissen stand im Zentrum, sondern «die Heranziehung sprachwissenschaftlicher Einsichten, Ergebnisse und Theorien zur Legitimation und Ausdifferenzierung anderweitig (z.B. pädagogisch) motivierter Konzepte» (Eichler \& Henze, 1990, S. 154). Sprechendes Beispiel für diese Tendenz war das, was als «kommunikative Wende» in die Geschichte des Deutschunterrichts eingegangen ist: die Ausrichtung der Lernziele und Lerninhalte auf die als oberstes Ziel gesetzte kommunikative Kompetenz. Hintergrund für diese Orientierung war eine Auffassung von Sprache und Sprachwissenschaft, die v. a. durch das Funkkolleg Sprache in den Siebzigerjahren weite Verbreitung fand. ${ }^{4}$ "Erziehung zur Kommunikationsfähigkeit» ist seither zu einem festen Bestandteil sprachdidaktischer Zielbestimmungen geworden.

Allerdings ist hier - gleichsam als Generalvorbehalt - eine Einschränkung zu machen: Sprachdidaktik ist ein weites Feld, das von der theorieverliebten Diskussion um Konzepte bis in die dicke Luft kleiner Schulstuben reicht. Was sich an Konzeptionen in der dünnen Luft der sprachdidaktischen Theoriediskussion ausmachen lässt, muss in der realen Organisation des Sprachunterrichts - in der Ausbildung von Lehrkräften und besonders im tatsächlichen Unterricht - nicht notgedrungen eine massgebliche Rolle spielen. 
Die «kommunikative Wende» war die letzte «Grosskonzeption» der Deutschdidaktik, auf die sich die unterschiedlichen Bereiche der Deutschdidaktik gemeinsam bezogen. Denn die «Zeit der Grossen Erzählungen» (Lyotard), der umfassenden programmatischen Neukonzeptionen war in den 80er-Jahren auch in der Sprachdidaktik vorbei. Allerdings lassen sich über den Zeitraum der letzten drei Jahrzehnte Entwicklungen nicht allein anhand von Konzeptdiskussionen verfolgen. Auch ein genuin didaktischer Blick erhellt interessante Zusammenhänge.

\section{Die sprachdidaktische Entwicklung aus der Perspektive des "didaktischen Dreiecks"}

An anderer Stelle (Sieber, 2003b, S. 128ff.) habe ich die Entwicklung der Diskussion anhand des klassischen «didaktischen Dreiecks» erläutert und dabei herausgestellt, wie sich das sprachdidaktische Interesse jeweils auf einen anderen Aspekt des Dreiecks konzentrierte:

- In den 60/70er-Jahren wurde in erster Linie die Achse Lehrer-Gegenstand diskutiert, erforscht und entwickelt - es war die grosse Zeit der Curriculumforschung, die mit dem Anspruch angetreten war, die Schule zu verbessern durch die "Revision des Curriculums» (S.B. Robinson). Die deutschdidaktische Diskussion war damals stark geprägt von den heftigen Debatten um die "Hessischen Rahmenrichtlinien» (Köhler \& Reuter, 1973).

- Während der 70/80er-Jahre kam dann eine andere Achse des didaktischen Dreiecks vermehrt in den Blick, denn zentrale Aspekte des Unterrichtens blieben auf der institutionellen Ebene des Curriculums ausgespart. So ist es nicht verwunderlich, dass als Nächstes stärker die konkreten Unterrichtssituationen zum Thema gemacht wurden: die Achse LehrerIn - SchülerIn. «Schülerorientierung» wurde zum Postulat. Diese fand in den Zielsetzungen und Konzepten einer «Erziehung zur Kommunikationsfähigkeit» mit starker Ausrichtung auf die Mündlichkeit ein breites Anwendungsfeld. In der Schreibdidaktik waren «Texte für Leser» (Boettcher, Firges, Sitta \& Tymister, ${ }^{5} 1978$ ) angesagt.

- In den 80/90er-Jahren - und gleichsam als Weiterentwicklung oder Ausdifferenzierung einer Schülerorientierung - rückte die Achse Schüler - Gegenstand ins Zentrum des Interesses. Die Fragen, die vermehrt die Welt im Kopf der Schüler im Fokus haben, hätten kaum so breit gestellt werden können, wenn nicht die kognitiven Wissenschaften, insbesondere die kognitive Psychologie, grosse Anstrengungen unternommen hätten, etwas Licht in die Verarbeitung von Erfahrungen, Eindrücken und Information zu bringen, was dann später oft als «kognitive Wende» charakterisiert wurde (Spinner, 1994). Lernerorientierung und Bilder von Lernenden standen also im Zentrum des Interesses. ${ }^{5}$ Unter dieser Perspektive wurde in der Schreibdidaktik eine enge Kooperation mit der Schreibforschung gesucht.

- In den späten 90er-Jahren ist zu beobachten, dass vermehrt das Umfeld, in dem das didaktische Dreieck situiert ist, in den Fokus der Aufmerksamkeit 
rückt: LehrerIn / SchülerIn / Gegenstand in der Schule als sozialem System, das selbst in soziale Kontexte eingebettet ist.

Es ist unschwer zu erkennen, dass die Ausweitung der Orientierungen von den Aspekten Lehrer / Schüler / Gegenstand auf die kulturelle Welt von den sprachdidaktischen Diskussionen um Mehrsprachigkeit, um Language Awareness oder um geschlechtstypischen Sprachgebrauch aufgenommen wurde. ${ }^{6}$ In der Schreibdidaktik führte diese Ausweitung zur Thematisierung der Literalitätsentwikklung innerhalb und ausserhalb der Schule. Dabei gewannen - gleichsam als Nebeneffekt - Fragen zur genaueren Konzeptualisierung von Textkompetenzen vermehrt Gewicht.

\section{Hinwendung zur Fundierung des Wissens durch Empirie}

Die beschriebene Ausweitung des Blicks aus dem didaktischen Dreieck auf das soziale und kulturelle Umfeld von Schule und Unterricht wurde durch eine weitere Tendenz massgeblich unterstützt: In der Fachdiskussion ist der Ruf nach einer Hinwendung zum tatsächlichen Unterrichtsgeschehen bereits eine alte Forderung, eine Hinwendung zu dem, was mit Unterricht konkret erreicht wird und was nicht - und bei wem es erreicht wird und bei wem nicht. Unter Stichworten wie «erfahrungswissenschaftliche Didaktik» (so Müller-Michaels, 1994, S. 40), "empirische Sprachdidaktik» (so Becker-Mrotzek, 1997b, S. 21) oder mit der Rede vom «Backen kleinerer Brötchen»(so Bremerich-Vos, 1993) ist die Forderung nach einer Hinwendung zur Empirie auch im Rahmen der Symposien Deutschdidaktik breit diskutiert worden. ${ }^{7}$ So wurde das letzte Symposion in Lüneburg, das unter dem Tagungsthema «Deutschunterricht - empirisch» stand, mit folgender Presseerklärung abgeschlossen:

Die Zeit der grossen programmatischen Debatten über den Deutschunterricht ist vorbei. Die Wissenschaft will sich künftig stärker der Erforschung von Lernvoraussetzungen und-erfolgen widmen. Das kann als wesentliches Ergebnis der Zusammenkunft von 460 Wissenschaftlerinnen und Wissenschaftlern im Rahmen des 15. Internationalen Symposions Deutschdidaktik in Lüneburg festgehalten werden. (SDD Mitgliederbrief 9/2004, S. 1).

Diese breit abgestützte Orientierung wäre nicht möglich geworden, wenn nicht im letzten Jahrzehnt die zentrale Vermittlungsfunktion der Fachdidaktik zwischen den Beständen des Fachwissens, dem pädagogischen Wissen und dem Wissen aus der schulischen Situation auch vermehrt empirisch untersucht worden wäre. Und dies gilt nicht allein für die enge Verbindung von Schreibforschung und Schreibdidaktik, auch wenn es in diesem Bereich eine exemplarische und bereits längere Tradition gibt. Bettina Hurrelmann (1998) erläutert dies nebst der Schriftlichkeitsforschung - auch an weiteren exemplarischen Beispielen: der Erzählforschung, der Kinder- und Jugendliteraturforschung, der Lesesozialisations- und der Medienforschung. Sie weist nach, wie sich in den letzten 
Jahren «integrative Forschungs- und Lehrbereiche» herausgebildet haben, denen vieles gemeinsam ist:

Sie vermitteln (1) ein fachspezifisch «reiches» Bild des Gegenstandes, sie diskutieren (2) die "mediale Prägung» von Kommunikations- und Bildungsprozessen im Bereich von Sprache und Literatur, sie betonen (3) die Erwerbsperspektive, wobei sie davon ausgehen, dass die individuelle, entwicklungsspezifisch «eigensinnige» Aneignung von Kompetenzen nicht beliebig ist, sondern regelhaft erfolgt, und zwar in Auseinandersetzung mit kulturellen Ordnungsmustern und Erwartungsschemata des Sprach-, Literatur- und Mediengebrauchs, die zugleich ein Geriist bieten für die individuelle kognitive Entwicklung. Sie sind schliesslich (4) empirieorientiert. Forschungsfeld und Anwendungsbezug sind vor allem - aber nicht ausschliesslich - (5) Unterricht und Schule.

Ich sehe im Schnittpunkt der bezeichneten fïnf Dimensionen: fachspezifische Gegenstandsentfaltung, Medialitätsperspektive, Erwerbsperspektive, Empirieorientierung, Anwendungsbezug schon in der Theorie die fruchtbarsten Ergebnisse der Deutschdidaktik der letzten Jahre. (Hurrelmann, 1998, S. 31).

Als typisch für die gegenwärtigen Tendenzen der Deutschdidaktik kann also Folgendes herausgestellt werden:

- die Ausweitung des Blicks auf vielfältige Sprachverwendung: produktiv und rezeptiv, in unterschiedlichen Medien, in der Erst- und Zweitsprache, schulisch und ausserschulisch, mit Blick auf die stattfindenden Entwicklungsprozesse

- die empirische Basis für die Gewinnung von Daten, Hypothesen und Erkenntnissen, was eine Kooperation von unterschiedlichen Wissenschaften und Wissenschaftlern voraussetzt, sowie die Nutzung eines breiten Spektrums von empirischen (qualitativen und quantitativen) Methoden

- die Beteiligung von Lehrkräften, die der Schulrealität zu ihrem Recht verhelfen, also der Einbezug und die Rückbindung der Forschungs- und Entwikklungstätigkeiten an Lehrerinnen und Lehrer.

\section{Zur Situation in der (Deutsch-) Schweiz}

Die Deutschschweiz hatte zunächst - in den 70er- und frühen 80er-Jahren - nur mittelbar Anteil an den Diskussionen, wie sie im deutschsprachigen Ausland geführt wurden. Die seminaristisch organisierte Lehrerbildung bot wenig Klangraum für die Resonanz, geschweige denn für die Teilnahme an den heftig geführten Debatten ausserhalb der Deutschschweiz. Und dies trotz der aktiven Beteiligung von Schweizer Linguisten an den Auseinandersetzungen - insbesondere sind hier Hans Glinz und Horst Sitta zu nennen. Unter diesen Voraussetzungen kamen vorerst die neuen Überlegungen und Vorschläge nicht durch einen breiten fachdidaktischen Diskurs, sondern - gleichsam subkutan - über die Konzeption von Lehrmitteln ${ }^{8}$ in die deutschschweizerische Diskussion: 
Anteil an den Veränderungen haben nicht zuletzt die neuen Generationen von Sprachbüchern in der Deutschschweiz [...] Diesen Sprachbüchern liegen - bei aller Verschiedenheit von Schwerpunktsetzung und didaktischer Ausarbeitung in weiten Teilen ähnliche grundlegende Auffassungen von "Sprache» und "Sprachunterricht"9 zugrunde. In die gleiche Richtung weisen auch neuere Lehrplanformulierungen in der (Deutsch-)Schweiz. (Sieber, 1990, S. 7).

Nach dieser ersten Phase der Neuorientierung erhielt die fachdidaktische Diskussion in der Deutschschweiz Auftrieb durch zwei institutionelle Veränderungen:

- Im Rahmen der Aktivitäten der EDK-Ost wurde eine «Kerngruppe Deutsch» ins Leben gerufen, die durch Kurse, Tagungen und Publikationen (Beiträge zum Deutschunterricht I, 1984; Beiträge zum Deutschunterricht II, 1985; Ruf, $\left.{ }^{4} 1992\right)$ eine Verbreiterung des deutschdidaktischen Diskurses verfolgte. Aus diesen Aktivitäten heraus wurde 1999 das deutschschweizerische Forum Deutschdidaktik gegründet. ${ }^{10}$

- Die wissenschaftliche Gesellschaft der DeutschdidaktikerInnen - das Symposion Deutschdidaktik - führte 1994 die zweijährlich stattfindende Tagung erstmals ausserhalb Deutschlands an der Universität Zürich durch (Sieber \& Sitta, 1994). Die Thematik des Zürcher Symposions bündelte eine damals aktuelle und entwicklungsmächtige Diskussion um Konzepte des Lernens und Bilder von Lernenden im Zeichen der kognitiven Wende (Sieber, 1994b; Spinner, 1994). Damit gelang der Anschluss der deutschschweizerischen Aktivitäten an den internationalen Diskurs ${ }^{11}$ und umgekehrt nahm die deutschsprachige Diskussion vermehrt die Ansätze und Projekte der Deutschschweiz wahr. ${ }^{12}$

Nebst den Diskussionen im gesamten deutschsprachigen Raum - wie sie zum Beispiel im Rahmen des Symposion Deutschdidaktik ${ }^{13}$ und in den einschlägigen Fachzeitschriften geführt werden - hat sich seit den 80er Jahren auch ein spezifischer Diskurs in der Deutschschweiz entwickelt. Er wurde angeregt u.a durch Untersuchungen zur Diglossiesituation der Deutschschweiz. Die Diskussion um Mundart und Hochdeutsch in der Deutschschweiz und insbesondere in den Deutschschweizer Schulen, die in der Vergangenheit schon immer geführt wurde, konnte dabei durch empirische Daten versachlicht werden (Sieber, 1990; Sieber \& Sitta, 1986; Sitta, 1979; E. Werlen, 1993 - zum aktuellen Stand: I. Werlen, 2004; Bildungsdirektion des Kantons Zürich \& Pädagogische Hochschule Zürich, 2003). Dabei waren zwei Perspektiven wegleitend, die in der neueren deutschdidaktischen Diskussion insgesamt zentral geworden sind:

(1) Der Einbezug von spracherwerbstheoretischen Perspektiven zur Erhellung der Dialekt/Standardprobleme ermöglichte neue Sichtweisen auf den Aufbau und die Einschätzung der Sprachkompetenzen (Burger \& Häcki Buhofer, 1994; Sieber \& Sitta, 1986, Kap. 4; Stern, 31994). Hinzu kam, dass die soziodemogra- 
fischen Veränderungen, die einen grösseren Anteil an multikulturellen Klassen zur Folge hatten, das Dialekt/Standardproblem in den Rahmen der Diskussionen um monolinguale Schulen und um die Notwendigkeit und Chancen von Mehrsprachigkeit stellten. Die Ausrichtung auf spracherwerbstheoretische Aspekte der Deutschschweizer Sprachlernsituation hat den Blick frei gemacht für Modellierungen, wie sie die Zweitspracherwerbsforschung entwickelt hat. Dies ist unter den aktuellen Voraussetzungen der multikulturellen Zusammensetzung der Schulklassen für eine gemeinsame Orientierung des gesamten Sprachunterrichts (Lüdi et al., 1998) hilfreich und ebenso notwendig unter der auch europäisch geforderten Zielsetzung der funktionalen Mehrsprachigkeit (Europarat - Rat für Kulturelle Zusammenarbeit, 2001) und den darauf bezogenen Bemühungen der EDK (Eidgenössische Erziehungsdirektorenkonferenz, 2003) zur Sprachförderung.

(2) Die Diglossiesituation in der Deutschschweiz mit der deutlichen Trennung der medialen Verwendung der beiden Sprachformen - Dialekt für gesprochene Sprache, Standardsprache für das Geschriebene - hat zu einem frühen Zeitpunkt bereits auf das Spannungsfeld von Mündlichkeit und Schriftlichkeit aufmerksam gemacht, das aktuell unter konzeptionellen Fragestellungen produktiv diskutiert wird (siehe unten). Die Diskussion um den Einbezug neuer Medien konnte hier nahtlos anknüpfen. Geführt wurde diese Diskussion insbesondere im Rahmen und im Umfeld eines mehrjährigen Projektzusammenhangs - «Lernen im Kontext neuer Medien» -, der vom Schweizerischen Nationalfonds, Schwerpunktprogramm «Zukunft Schweiz» gefördert wurde. ${ }^{14}$

Darüber hinaus sind mit Forschungsprojekten aus der Deutschschweiz (z.B. Häcki Buhofer, 1985; Hornung, 2002; Nussbaumer, 1991; Portmann, 1991; Sieber, 1994a; Sieber, 1998a) in den 90er-Jahren Anknüpfungspunkte an die internationale Diskussion der Schreibforschung und Schreibdidaktik geschaffen worden. Diese Projekte haben sich an der fruchtbaren Zusammenarbeit von Schreibdidaktik und Schreibforschung beteiligt.

\section{Schreibforschung und Schreibdidaktik - eine produktive Kooperation}

Schreiben lernen als eine der zentralen Aufgaben des Erstsprachunterrichts ist in den letzten beiden Jahrzehnten im deutschsprachigen Raum breit diskutiert worden. Dabei konnte und kann die Diskussion von einer engen Beziehung zwischen Schreibforschung und Schreibdidaktik profitieren. Helmuth Feilke und Paul Portmann kommentieren in ihrem Sammelband mit dem bezeichnenden Titel «Schreiben im Umbruch - Schreibforschung und schulisches Schreiben» die Situation in der zweiten Hälfte der Neunzigerjahre so: 
Die Diskussion um das Schreiben im Unterricht kann sich heute - anders als noch vor zehn, fünfzehn Jahren - auf ein umfangreiches, empirisch abgestütztes Wissen über Schreibprozesse, Schreiblernen und Texte berufen. Auch wenn unsere Einsichten in die entscheidenden Zusammenhänge noch viele Lücken aufweisendie vorhandenen Forschungsergebnisse erlauben es auf ganz neue und folgenreiche Weise, die Vorgänge beim Schreiben zu konzeptualisieren und mit Fragen der Schreibdidaktik zu verknüpfen. (Feilke \& Portmann, 1996, S. 14).

Seit den 80er-Jahren ist in der deutschdidaktischen Diskussion - nach einer Dekade der Mündlichkeit mit der Favorisierung des Lernziels «Kommunikationsfähigkeit» - das Schreiben neu entdeckt worden. Massgeblich dazu beigetragen haben kulturgeschichtliche und -wissenschaftliche Untersuchungen $\mathrm{zu}$ «Oralität und Literalität - die Technologisierung des Wortes» (so die deutsche Übersetzung von Ong, 1982/1987). Ebenso haben sprachtheoretische Klärungen, wie sie von Koch und Oesterreicher (1985) und Koch und Oesterreicher (1994) unter den Aspekten Sprache der Nähe / Sprache der Distanz vorgelegt worden sind, zu einer breiten Diskussion über konzeptionelle Mündlichkeit und konzeptionelle Schriftlichkeit geführt. Die Aufnahme dieser Diskussionen im deutschdidaktischen Diskurs (z.B. Bachmann, 2002; Feilke, 2002; Sieber, 2000) hat Fragen der Literalität - und unter ästhetischen und literaturdidaktischen Gesichtspunkten der "Literarität» (z.B. - und wegweisend - Dehn, 1999) - ins Zentrum schreibdidaktischer Überlegungen gerückt.

Aus der Fülle der Aspekte sollen im Folgenden drei herausgehoben werden, die zentrale Momente der aktuellen Beschäftigung mit Schreiben betreffen:

- die Modellierung der Schreibhandlungen als komplexe (Problemlösungs-) Prozesse

- der Einbezug von Entwicklungsprozessen zur Erklärung des Aufbaus von Schreibfähigkeiten

- die aktuelle Fassung der schreibdidaktischen Bemühungen unter dem Stichwort der "prozessorientierten Schreibdidaktik» und die Konsequenzen, die sich daraus für die Schreibförderung ergeben.

\section{Schreiben als komplexe Tätigkeit}

Schreiben ist eine komplexe Tätigkeit. Ludwig (1995) weist in seiner «Skizze zu einer Theorie des Schreibens» darauf hin, dass wir (noch) über keinen Begriff des Schreibens verfügen, "der alle Vorstellungen, Auffassungen und Praxen von Schreiben, die im Verlauf der Geschichte zutage getreten oder überhaupt möglich sind, erfassen könnte.» (Ludwig, 1995, S. 275). In einem neueren Versuch (Ludwig, 2003a) arbeitet er für die «Herstellung einer schriftlichen Kommunikation» insgesamt fünf verschiedene Konzeptionen heraus und stellt das Schreiben als Produktion von Texten als aktuell wichtigste Konzeption dar:

Wenn in der modernen Schreibforschung von "Schreiben" die Rede ist, dann setzt man wie selbstverständlich voraus, dass es sich um das Schreiben von Texten han- 
delt. (....) So stellt sich die Frage, wie das Texteschreiben als Handlung zu beschreiben ist und in welchem Verhältnis es zu den anderen Konzeptionen des Schreibens steht. (Ludwig, 2003a, S. 11).

Diese Bestimmung des Texteschreibens wäre nicht möglich geworden, wenn nicht auf eine reiche Fülle von Untersuchungen und Modellierungen der Schreibforschung hätte zurückgegriffen werden können.

Einen grossen Entwicklungsschub erhielt die Schreibforschung ab den 80er-Jahren, als Schreiben unter kognitionswissenschaftlichen Perspektiven zum Gegenstand intensiver Forschungstätigkeiten geworden war. In der ersten "grossen Phase» der Schreibforschung wurde Schreiben als problemlösendes Handeln modelliert und in Beziehung zu anderen Problemlöseaktivitäten gesetzt. Dabei wurden v.a. Prozesse der Planung und Revision in den Vordergrund gerückt. Grundlegend dafür waren die Arbeiten und Modellkonzeptionen von Hayes und Flower (1980), Flower und Hayes (1980). (Zusammenfassend dazu: Bachmann, 2002, S. 28-55.; Molitor-Lübbert, 1996, S. 1005-1008; Sieber, 2003a, S. 212-216). In der schweizerischen Diskussion wurden diese Modelle u.a. ausdifferenziert zum "Orchestermodell der Textproduktion» (Baer, Fuchs, ReberWyss, Jurt \& Nussbaum, 1995).

Diese kognitionspsychologisch ausgerichteten Untersuchungen orientierten sich fast ausschliesslich an Experten-Modellen und rückten die Planungsprozesse des Schreibens in den Mittelpunkt. Das führte u.a. zu didaktischen Modellen, welche die Planung vor dem Schreiben stark aufwerteten und diese Strategie als notwendige Bedingung für gelingendes Schreiben setzten. Eine fundamentale Kritik an den Prozessmodellen formuliert Ortner (2000, S. 97ff.), indem er der Schreibforschung generell und den am Problemlöseprozess orientierten Ansätzen speziell eine Orientierung an repräsentationistischen Zweck-Mittel-Schemata vorwirft:

Die gesamte Schreibforschung ist finalistisch-instrumentalistisch infiziert. Sie steht ganz im Bann des Zweck-Mittel-Schemas: die Sprache als Werkzeug, um die Intentionen, Gedanken usw. zu realisieren, und die Äusserung/der Text als Repräsentanten dieser Intentionen, Gedanken usw. (Ortner, 2000, S. 97).

Ortner schlägt demgegenüber eine Orientierung an Strategien als «erworbene Ablauf- und Organisationsschemata» (Ortner 2000, S. 351) vor, um der Komplexität des Schreibprozesses - und damit einer wirksamen schulischen Schreibförderung - näher zu kommen. Diese Kritik ist in die Diskussion um «Prozesse, Prozeduren und Produkte» (so der Untertitel von Baurmann \& Weingarten, 1995) im Rahmen einer "prozessorientierten Schreibdidaktik» aufgenommen worden. 
Entwicklung der Schreibfähigkeiten - Erwerbsperspektive Nebst dem Prozess gelangte auch die Erwerbsperspektive von Schreibfähigkeiten in den Fokus der Aufmerksamkeit. Grundlegend war hier das Modell der Schreibentwicklung, wie es Bereiter (1980) und Bereiter und Scardamalia (1987) vorgelegt haben (zusammenfassend: Bachmann, 2002, S. 43-48; Molitor-Lübbert, 1996, S. 1010-1016; Sieber, 2003a, S. 216-219).

Bereiter (1980) unterscheidet die folgenden Fähigkeiten:

- Schreiber schreibt, solange ihm etwas einfällt (Associative Writing).

- Schreiber befolgt schulische Konventionen (Performative Writing).

- Schreiber orientiert sich auf einen potentiellen Leser hin (Communicative Writing).

- Schreiber beurteilt sein Produkt als Leser (Unified Writing).

- Schreiber schreibt zur (eigenen) Wissensgewinnung (Epistemic Writing).

Neuere Modelle (z.B. Becker-Mrotzek, 1997, S. 294-306) gehen von einzelnen Dimensionen des Schreibens aus, die unterschieden werden müssen: die Sachverhalte im Text (Was schreiben?), die textuellen Realisierungen (Wie schreiben?), die Organisation des Schreibprozesses (Wie organisieren?). Bereits Flower und Hayes (1980) haben dafür das Bild des Jongleurs geprägt, der mit den unterschiedlichen Ansprüchen an das Schreiben jonglieren muss.

Die einzelnen Dimensionen entfalten sich im Laufe der ontogenetischen Entwicklung. Die Entwicklung beginnt jeweils bei den Kernen der Dimensionen und setzt sich - unterstützt durch die Ausbildung von Routinen - zu den Rändern hin fort. Die Entwicklung der Schreibfähigkeiten ist abhängig vom Lernalter, d.h. von den (Schreib-)Erfahrungen und nicht allein vom Lebensalter. Die Entwicklung zieht sich - bei Gebrauch - ins Erwachsenenalter hin weiter und ist wohl niemals abgeschlossen. Die Entwicklung der Schreibfähigkeiten ist also kein linearer Prozess einer stetigen Zunahme von Fähigkeiten. Vielmehr lässt sich u.a. Folgendes beobachten:

- eine altersmässige Zunahme quantitativ, aber auch von lokalen zu globalen Strukturen. («Von der Reihung zur Gestaltung», wie der Titel von Augst und Faigel (1986) zusammenfasst).

- Planungsveränderungen von der Oberfläche zur Textstruktur und zum Inhalt.

- Überarbeitungen zeitigen erst mit zunehmendem Alter Textverbesserungen.

- Wichtige Entwicklungsschritte erfolgen erst im Übergang vom Jugendalter zum jungen Erwachsenenalter (16-18 Jahre), also erst nach der obligatorischen Schulzeit.

- Immer ist aber auch mit «der Gleichzeitigkeit des Ungleichzeitigen» (Bachmann, 2002, S. 251) zu rechnen. 
Mit dem Blick auf den Erwerb von Schreibfähigkeiten werden die Expertenmodelle durch weitere Aspekte angereichert. Dazu gehört der entstehende Text mit seinen Ansprüchen ebenso wie die Schreibsituation und die verschiedenen Strategien, die von unterschiedlichen Schreibern aufgebaut und verwendet werden. Anfänglich war die Erforschung der Strategien, die Schreiber anwenden, stark auf die Planung vor dem Schreiben ausgerichtet. Unterdessen wissen wir, dass unterschiedliche Strategien zu erfolgreichem Schreiben führen können.

Insbesondere Ortner schlägt - aus der Kritik an den reduktionistischen Prozessmodellen - eine Auseinandersetzung mit Strategien des (erfolgreichen) Schreibens vor. Er unterscheidet in einer materialreich angelegten Beschreibung insgesamt 10 Strategien:

(1) (Scheinbar) nicht zerlegendes Schreiben. Schreiben in einem Zug. Schreiben im Stil der pensée parlée, écriture automatique. Typ des Aus-demBauch-heraus-(=Flow-)Schreibers.

(2) Einen Text zu einer Idee schreiben.

(3) Schreiben von Textrevisionen zu einer Idee.

(4) Herstellen von Texten über die redaktionelle Arbeit an Texten (Vorfassungen), von verbesserten Versionen durch Arbeit am vorliegenden Text.

(5) Planendes Schreiben (Plan = eine Version in Kurzschrift).

(6) Einfälle ausserhalb eines Textes weiterentwickeln. Konzeptuell extralingual + niederschreibend.

(7) Schrittweises Vorgehen - der Produktionslogik folgend.

(8) Synkretistisch-schrittweises Schreiben.

(9) Moderat produktzerlegend. Das Schreiben von Produktsegmenten.

(10) Schreiben nach dem Puzzle-Prinzip. Extrem produktzerlegend. (Ortner, 2000, S. VI und S. 346-564).

In einfacherer, aber auch entsprechend weniger ausgreifender und differenzierter Weise hat Barton (1994) mit Hinweis auf Chandler (1992, S. 57-60) vier Schreibstrategien zur Unterscheidung der "Ablauf- und Organisationsschemata» vorgeschlagen:

"The oil painting strategy: the, typical Discoverer strategy of doing minimal preplanning, jotting down ideas as they occur and reworking the text repeatedly".

The architectural strategy : which involves conscious planning and organization with only limited drafting and reviewing".

The bricklaying strategy:,polishing each sentence before proceeding to the next».

The watercolour strategy: an attempt to produce a complete version relatively rapidly».»(Barton, 1994, S. 173f.).

Jede Strategie kann zum Ziel führen, nicht jede muss für alle Schreibanlässe optimal sein. Und: Nicht alle Schreiber haben Zugang zu jeder Strategie - es gibt also nicht nur ein Modell, wie man schreiben kann! Damit wird für das Schreiben-Lernen der Austausch von Schreiberfahrungen wichtig, wie er im Reden 
über Texte und deren Qualitäten gefordert wird (Nussbaumer \& Sieber, 1995) und wie dies z.B. mit didaktischen Konzepten von «Schreibkonferenzen» (Spitta, 1992) oder der "Sequenzierung des Schreibprozesses» (Fix, 2000) umzusetzen versucht wird.

Die Darstellung der Strategiediskussion macht deutlich, dass wir insgesamt noch weit davon entfernt sind, über eine brauchbare Modellierung des Schreibprozesses zu verfügen, die sowohl entwicklungs- wie auch kontextsensitiv ist. Doch liegen viele Elemente vor, die eine wirkungsvolle Unterstützung des Schreibenlernens ermöglichen. Sie werden in jüngerer Zeit unter dem Stichwort "prozessorientierte Schreibdidaktik» gefasst.

\section{Schreibförderung in einer prozessorientierten Schreibdidaktik}

Was sich aus der Zusammenarbeit zwischen Schreibforschung und Schreibdidaktik in den letzten Jahrzehnten an Konzepten für das Schreibenlernen entwikkelt hat, wird unter dem Stichwort «prozessorientierte Schreibdidaktik» oder "prozessorientierter Schreibunterricht» zusammengefasst (z.B. Baurmann, 2002; Baurmann, 2003; Ludwig, 2003b; Portmann-Tselikas, 2005).

Baurmann nennt drei Merkmale, die die Prozessorientierung des Schreibens auszeichnen:

Die Theorie und Praxis des Schreibens in der Schule bezeichnet man - erstens dann als prozessorientiert, wenn das Verfassen von Texten als komplexer Vorgang verstanden wird, dessen Teilprozesse im Unterricht nachhaltig beachtet werden. Bei der Textproduktion lassen sich Teilprozesse unterscheiden - etwa konzeptionelle Prozesse, Vorgänge des Formulierens, des Ausführens und Inskribierens sowie des Überprüfens und Überarbeitens. (...) Die einzelnen Teilprozesse verlaufen dabei nicht ausschliesslich und streng sukzessiv, sondern pendeln interaktiv zwischen verschiedenen Ebenen hin und her; sie sind jederzeit wiederholbar und ausserdem auf sich selbst beziehbar.

Schlüssig eröffnen sich - zweitens - aus einer solchen Sicht des Gegenstandes Möglichkeiten, die gesamte Unterrichtsgestaltung prozessorientiert anzulegen. (...) Innerhalb eines so konturierten Unterrichts wird es möglich, Schreiben und die schriftliche Reflexion dieser Tätigkeiten über die gegenwärtige Praxis hinaus erheblich auszuweiten. Das Schreiben nach Mustern wird dann neben dem Verfassen epischer Kurzformen auch das Schreiben nach umfangreicheren Vorlagen einschliessen (etwa das Verfassen eigener Western und Liebesromane und das Nachdenken über solche Textsorten). (....)

Über die Grundentscheidung und die Unterrichtsgestaltung hinaus ergeben sich - drittens - an einem weiteren bedeutsamen Punkt Möglichkeiten, das Schreiben in der Schule prozessorientiert zu fassen - nämlich hinsichtlich der curricularen Planung. Dabei zielen die curricularen Erwägungen darauf ab, Schülern auf möglichst vielfältige und anregende Weise Zugänge zu verschiedenen Schreib- 
funktionen zu eröffnen und sie zu erfolgversprechenden Schreibstrategien zu ermutigen. (Baurmann, 2003, S. 254f.).

Mit der prozessorientierten Schreibdidaktik liegt eine reiche Palette von fundierten Überlegungen und Anregungen vor, die durch eine Integration der vielfältigen Perspektiven, wie sie die Schreibforschung ausdifferenziert hat, Schreibunterricht gestaltbar macht. So kann die Schule ihre Aufgabe einer verstärkten Schreibförderung professionell und wirkungsvoll wahrnehmen.

An anderer Stelle (Sieber, 2003a, S. 219f.) habe ich selbst eine Skizze der Schreibförderung entwickelt, die drei Säulen der Schreibförderung unterscheidet: Schreibprozesse, Überarbeitungsprozesse und Beurteilungsprozesse.

\section{Tabelle 1: Ein Drei-Säulen-Modell der Schreibförderung (Sieber, 2003a, S. 219)}

\begin{tabular}{|l|l|l|}
\hline \multicolumn{1}{|c|}{ Schreibprozesse } & \multicolumn{1}{|c|}{ Überarbeitungsprozesse } & \multicolumn{1}{c|}{ Beurteilungsprozesse } \\
\hline $\begin{array}{l}\text { Kare Vorstellungen über den } \\
\text { Schreibanlass entwickeln }\end{array}$ & Abstand zum Text gewinnen & $\begin{array}{l}\text { Texte von andern } \\
\text { zur Kenntnis nehmen }\end{array}$ \\
\hline $\begin{array}{l}\text { Sammeln und planen von } \\
\text { Inhalten }\end{array}$ & Dissonanzen entdecken & $\begin{array}{l}\text { Über Textqualitäten } \\
\text { reden lernen }\end{array}$ \\
\hline $\begin{array}{l}\text { Schreibprozesse mehrphasig } \\
\text { anlegen }\end{array}$ & Alternativen finden & $\begin{array}{l}\text { Von der Fremd- zur } \\
\text { Selbstbeurteilung }\end{array}$ \\
\hline
\end{tabular}

Dieses «Drei-Säulen-Modell der Schreibförderung» kann auch verdeutlichen, in welchen Bereichen besonderer Bedarf an Weiterentwicklung besteht: Für die erste Säule (die Anregung von Schreibprozessen) sind in der Sprachdidaktik viele Erfahrungen und Anregungen greifbar. ${ }^{15}$ Und auch das Feld der Überarbeitungen ist bereits hilfreich bearbeitet. ${ }^{16}$ Hingegen ist bei der dritten Säule, den Beurteilungsprozessen, noch vieles zu tun - insbesondere dort, wo es um den Aufbau von Selbstbeurteilungskompetenz hinsichtlich der Textqualitäten geht.

\section{Aktuelle Tendenzen}

Um aktuelle Entwicklungen innerhalb der Deutschdidaktik zu charakterisieren, sollen abschliessend zwei Problembereiche herausgestellt werden. Sie betreffen einerseits die Veränderung der Orientierung des Bildungssystems und der Bildungsdiskussion - weg von den Zielen und Inhalten und hin zu den erreichten Effekten. Die aktuellen Diskussionen um Bildungsstandards und Kompetenzen machen dies deutlich.

Zum andern lässt sich im Licht der Literalitätsdebatte - und nicht unabhängig von ihr - eine über die Prozessorientierung hinausgehende Modellierung der Schreibentwicklung beobachten, die die Eigengesetzlichkeit der Textgenese und 
der Textkompetenz stärker in Betracht zieht und damit zu einer umfassenderen Sichtweise des Aufbaus von Schreibkompetenzen in einer reichen literalen Umwelt gelangt.

\section{Wahrnehmung und Orientierung an den erzielten Effekten}

Auch wenn ein politisches Phänomen am Beginn der markanten Entwicklung der Schreibforschung stand - der Sputnik-Schock (Ludwig, 2001) -, so haben sich zumindest im deutschsprachigen Raum die Debatten weitgehend im innerwissenschaftlichen Kreis bewegt. Das hat sich mit der öffentlichen Thematisierung von schulischen Leistungen, wie sie anhand von internationalen Vergleichsstudien (wie TIMMS, PISA, ALL) breit diskutiert worden sind, grundlegend gewandelt. Kommt dazu, dass auch Aspekte des Bildungsmanagements und der Qualitätssicherung und Evaluation Perspektiven auf Schulentwicklung ermöglichen, die sich nicht mehr vor dem Hintergrund des didaktischen Dreiecks fassen lassen.

Im deutschdidaktischen Diskurs waren lange Zeit die Ziele und Inhalte, die Lehrer-Schüler-Beziehung und die Beziehung zwischen Lernenden und Gegenstand - also die klassischen Elemente des didaktischen Dreiecks - weit wichtiger als die Ergebnisse und Effekte, die Leistungen und Mängel, die als Resultate der schulischen Bemühungen beim Abschluss der Schullaufbahn festzustellen sind.

Ein Sprengen dieses Korsetts hat die Verlagerung vom Interesse am Input hin zu den Lernergebnissen, die Ausrichtung auf im schlechteren Fall den Output und im besseren Fall das Outcome des Systems mit sich gebracht. (Ich wähle hier bewusst die ökonomische Terminologie, die eine sinnvolle Unterscheidung macht zwischen dem Output als Leistung eines Systems und dem Outcome als Wirkung des Outputs, als Summe an erwünschten und unerwünschten Effekten und Leistungen.)

Unter dieser Perspektive sind nicht mehr in erster Linie die Bildungsprogramme der Schulen für die Definition des Bildungserfolgs wichtig, sondern die tatsächlichen Effekte, die diese Programme hervorrufen. Das steht zur Art und Weise, wie der Bildungsdiskurs meist geführt wird, ziemlich quer. Dies hat Jürgen Oelkers schon vor Jahren bündig so auf den Punkt gebracht: «Traditionell ist Bildung wesentlich gute Absicht und nicht Orientierung am Resultat.» (Jürgen Oelkers in: NZZ am Sonntag, 1. September 2002, S. 19).

Eine solche Sichtweise kann auch entlastend wirken: Die Schule kann sich - gerade in der Literalitätsförderung - von einer bildungsbürgerlich geprägten Sicht auf Bildungsbedürfnisse lösen und sich der Aufgabe stellen, die für eine Wissensgesellschaft notwendigen Sprachfähigkeiten für alle Mitglieder der Gesellschaft zu entwickeln. Denn noch zu keiner Zeit war Sprachförderung so nötig und so wichtig wie in der Gegenwart, niemals bisher sahen sich so viele Menschen so hohen sprachlichen Anforderungen gegenüber wie heute. Dazu reicht allein die Diskussion um Bildung nicht aus. Denn - wie es in einer immer noch bemerkenswerten und klärenden Formulierung heisst: 
Bildung ist kultivierter Geschmack und am Objekt geschulte Urteilskraft. Beides sind Lernaufgaben des Lebens und nicht der Schule. Wenn es gut geht, dann ist Schulbildung dabei eine Hilfe und kein Hindernis, aber es wäre fatal, Bildung einfach mit Schulerfahrung gleichzusetzen. (...) Nicht ohne Grund war die klassische Theorie der Bildung immer schulkritisch und sind Bildungsromane immer gleichbedeutend gewesen mit der Befreiung von den Zumutungen der Schulerfahrungen. Demgegenüber verfolgen staatliche Schulen pragmatische Zwecke, die mit universellen Erwartungen der allgemeinen Menschenbildung nicht sehr weit in Einklang gebracht werden können. (J. Oelkers in: Die Zeit, Nr. 27; 27. Juni 2002, S. 36).

So ist denn auch die Sprachförderung nur dann wirkungsvoll, wenn sie sich auf verbindliche Standards hin ausrichten kann, deren Überprüfung möglich gemacht wird, damit Bildungsbemühungen nicht gute Absicht bleiben, sondern sich an den Resultaten, an den Effekten orientieren können. Hier sind denn auch die Bemühungen, wie sie z.B. mit dem EDK-Projekt HarmoS (EDK, 2004) verfolgt werden, zu begrüssen.

Die Orientierung am Outcome, an den erzielten gewünschten und nicht intendierten Effekten hat eine aktuelle Debatte im Hinblick auf Literalität ins Zentrum der Aufmerksamkeit gerückt: Wie ist die Sicherung und Weiterentwicklung einer literalen Gesellschaft zu gewährleisten, wenn immer mehr Menschen über höhere literale Kompetenzen (rezeptiv im Lesen und produktiv im Schreiben) verfügen müssen, damit ein lebenswertes Leben in unseren Gesellschaften gewährleistet ist? Wie ist dies zu erreichen angesichts der Tatsache, dass die empirischen Befunde - aus den internationalen Untersuchungen wie IALS, PISA, ALL - deutlich das aktuelle Verfehlen dieses Ziels belegen?

\section{Literalitätsdebatte und Schreibförderung - Aufbau von Textkompetenz}

Unter den spezifischen Bedingungen der Deutschschweizer Diskussion wurde oben herausgestellt, dass die Dialekt/Standard-Situation die Unterschiede zwischen (konzeptioneller) Mündlichkeit und Schriftlichkeit in besonderer Weise erfahrbar und einsichtig macht. Deshalb ist die Aufmerksamkeit auf den Aufbau von konzeptioneller Schriftlichkeit - was nach wie vor eines der grundlegenden Ziele der schulischen Sprachförderung darstellt - hier besonders ausgeprägt. So wurden schon in frühen Arbeiten (Sieber \& Sitta, 1986, Kap. 5) die grundsätzlichen Unterschiede zwischen gesprochener und geschriebener Sprache herausgearbeitet. Sie wurden dann in weiteren Arbeiten differenziert. ${ }^{17}$

Die aktuelle Diskussion behandelt diese Problematik unter verschiedenen Stichworten - mit Bezug auf unterschiedliche Referenzen.

Feilke $(2002 ; 2003)$ argumentiert aus einer sprachtheoretischen Position mit Blick auf «schriftlich-konzeptuale» Fähigkeiten und fasst als Ideal «konzeptio- 
neller Literalität», was als «idealisierte Norm der Schriftlichkeit» (Feilke, 2003, S. 179) angesehen werden kann. Sie trägt die Merkmale der Distanzsprache (Koch \& Österreicher, 1994):

Nähe und Distanz kann es medial sowohl mündlich als auch schriftlich geben. Erst wenn Schrift funktional auf sozial distante und abstrakte Adressaten (z.B. ein allgemeines Publikum) sowie auf zeitlich und räumlich zerdehnte Kommunikation (Ehlich) bezogen wird, entfaltet das sprachliche Handeln die Potentiale konzeptioneller Literalität. (Feilke, 2003, S. 179).

Die vorgestellte Zielnorm konzeptioneller Schriftlichkeit orientiert sich also an einer maximal kontextentbundenen Kommunikation.

Einen anderen Zugang zur gleichen Problematik - geprägt auch durch Erfahrungen mit fremdsprachigen Lernenden - wählt Portmann-Tselikas (2002; 2005). Er fordert eine Einbettung prozessorientierter Vorschläge in einen weiteren Rahmen der «sozial geprägten Formen des literalen Denkens und des schriftlich geprägten Sprachgebrauchs» (Portmann, 2005, S. 174). Er geht aus von einer Differenzierung der Sprachkompetenz und nimmt die in der sprachlichen Realisierung feststellbaren Unterschiede unter der Perspektive einer textuellen Kompetenz auf. Portmann-Tselikas (2002, S. 14) versteht unter Textkompetenz zunächst "die Fähigkeit, mit Texten rezeptiv und produktiv umzugehen», um dann den Begriff genauer so zu fassen:

"Textkompetenz umfasst jene Schemata, Strategien und Techniken, die nötig sind, um den mit textueller Kommunikation verbundenen Aufgaben gerecht zu werden.» (Portmann-Tselikas, 2002, S. 15).

Basis für diese Textkompetenz ist eine spezifische Ausformung der allgemeinen Interaktionsfähigkeit, die sich durch mindestens vier Eigenschaften auszeichnet: ein Höchstmass an Sprachlichkeit, Kontextreduktion, Themenzentriertheit sowie Strukturiertheit und Formbestimmtheit.

Nicht zufällig sind in diesen Eigenschaften wesentliche Merkmale enthalten, die auch «konzeptionelle Schriftlichkeit» auszeichnen. Sie weisen zusätzlich auf einen weiteren Differenzierungsversuch hin: Portmann-Tselikas (2002, S. 16) bemerkt selbst die grosse Affinität, welche seine Definition von Textkompetenz mit einer Unterscheidung hat, die Cummins (Cummins, 1980; 1991; 2004) eingeführt hat. Cummins unterscheidet eine allägliche Kommunikationsfähigkeit (BICS - «basic interpersonal communication skills») von einer kognitivakademischen Sprachkompetenz (CALP - «cognitive-academic language proficiency»).

Die Schreibdidaktik muss sich also um den Aufbau von konzeptioneller Literalität (Feilke), von kognitiv-akademischer Sprachkompetenz (Cummins), von 
Textkompetenz (Portmann) kümmern. Allen drei Versuchen ist gemeinsam, dass sie in der Ausbildung einer spezifischen «kommunikativen Grundhaltung der Schriftlichkeit» (Sieber, 1998a, S. 188) ein zentrales Ziel der schulischen Schreibförderung sehen. Dieser Zielsetzung stehen aktuelle Sprachentwicklungen entgegen, die eine Orientierung an konzeptioneller Mündlichkeit, an einer «Sprache der Nähe» favorisieren. Dies zeigt sich nicht allein im Zusammenhang mit der Schriftlichkeit in neuen Medien (z.B. Dürscheid, 2003), es verweist ebenso auf längerfristige Entwicklungstendenzen der Angleichung geschriebener an gesprochene Sprache (zusammenfassend: Sieber, 1998a; 2000). Kommt hinzu, dass das Ziel entwickelter Schreibkompetenz kaum allein mit schulischer Schreibförderung zu erreichen ist. Zumindest weisen aktuelle Ergebnisse auf einen engen Zusammenhang zwischen entwickelter Schreibkompetenz und reicher literaler Erfahrung hin. Unsere Untersuchungen Jugendlicher (Bachmann \& Sieber, 2004) zeigen, dass für die Ausbildung voraussetzungsreicher schriftsprachlicher Fähigkeiten der Schule allein enge Grenzen gesetzt sind. Wir haben das zusammenfassend so formuliert:

Für die Entwicklung komplexer literaler Kompetenzen ist die motivierte ausserschulische literale Praxis unverzichtbar. Eine einseitig auf das Lesen und Schreiben in der Schule gerichtete literale Motivation unterstützt lediglich die Ausdifferenzierung vergleichsweise wenig anspruchsvoller literaler Kompetenzen. (Bachmann, Bertschi-Kaufmann, Kassis, Schneider \& Sieber, 2004, S. 242).

Um voraussetzungsreiche literale Kompetenzen aufzubauen, ist offenbar nebst der schulischen Arbeit an Texten auch eine ausserschulische literale Praxis notwendig, die über Lektüren eine zunächst unbewusste Kenntnisnahme von textuellen Realisierungsformen und Kontexten ermöglicht, die sich erst später in der aktiven Produktion von Texten zeigt. Eine solche reiche literale Praxis kann die vielfältigen Modelle konzeptioneller Schriftlichkeit zunächst rezeptiv zugänglich machen, damit sie später auch produktiv in der eigenen Schreibpraxis genutzt und ausdifferenziert werden können.

Das sind keine geringen Herausforderungen - und sie können von der Schule allein nicht bewältigt werden: "Gelingende literale Sozialisation ist über die Schule hinaus auf sozialpolitische Unterstützung angewiesen.» (Bachmann, Bertschi-Kaufmann, Kassis, Schneider \& Sieber, 2004, S. 244).

Die Schule kann aber einen wesentlichen Beitrag dazu leisten, indem sie ein reiches Feld von literalen Erfahrungs- und Betätigungsmöglichkeiten bereitstellt und die Lernenden in ihrem Prozess der literalen Entwicklung begleitet und unterstützt. Denn: Schreiben ist lernbar - in reichhaltigen und vielfältigen Situationen, in häufiger aktiver und reflektierter Auseinandersetzung und in einem literalitätsfördernden Klima, das schulische und ausserschulische Erfahrungen einbezieht. 


\section{Anmerkungen}

1 In einer früheren Arbeit wurden diese Bezüge insbesondere aus der Perspektive von Sprachwissenschaft und Sprachdidaktik als ein Verhältnis zweier ungleicher Schwestern dargestellt (Sieber, 1998b).

2 Der Teil III dieses Handbuchs Schreiben umfasst auf den Seiten 171-270 folgende Artikel: Geschichte der Didaktik des Texteschreibens (Otto Ludwig, S. 171-177), Entwicklung schriftlich-konzeptualer Fähigkeiten (Helmuth Feilke, S. 178-192), Entwicklung schriftlich-konzeptualer Fähigkeiten im mehrsprachigen Kontext (Margarete Ott, S. 193-207), Modelle des Schreibprozesses (Peter Sieber, S. 208-223), Formen schriftlicher Texte (Eduard Haueis, S. 224-236), Stil und Stilistik (Ulf Abraham, S. 237-248), Schreiben und neue Medien (Ulrich Schmitz, S. 249-260), Schreibschwierigkeiten (Iris Füssenich, S. 261-270).

3 Detailliertere Beschreibungen der Fachgeschichte sind greifbar in Fingerhut (1995); Becker-Mrotzek (1997b); Sieber (1998b); Hurrelmann (1998); Glinz (2003); Kämper-van den Boogaart (2003).

4 Paradigmatisch wird diese Sichtweise in der Einleitung des Funkkollegs «Sprache» erläutert: "Sprache wird als Instrument der Kommunikation und als sozial vereinbartes System von Zeichen behandelt. Andere mögliche Standpunkte, etwa: die Sprache als einen geschichtlichen Prozess oder als ein geschichtliches Produkt, als Kulturgut, als Ausdruck einer Mentalität oder gar als jeweilige Gliederung der Wirklichkeit aufzufassen, werden im folgenden ausser Acht gelassen. Damit konzentrieren wir unsere Einführung auf den inhaltlichen und methodischen Bereich, unter dem heute die moderne Linguistik allgemein verstanden wird.» (Baumgärtner, Fritz, Herrlitz, Hundsnurscher, Kastovsky et al., 1973, S. 18).

5 So ist es denn auch kein Zufall, dass das 10. Symposion Deutschdidaktik 1994 in Zürich unter dem Motto: «Konzepte des Lernens - Bilder von Lernenden» stand (Sieber, 1994b; Spinner, 1994).

6 Exemplarisch wird diese Ausweitung sichtbar im Konzept und Titel des Bandes von Linke und Oomen-Welke (1995). Herkunft, Geschlecht und Deutschunterricht: oben - unten / von hier - von anderswo / männlich - weiblich.

7 Während das Freiburger Symposion Deutschdidaktik 2000 dem Verhältnis von empirischer Unterrichtsforschung und Deutschdidaktik schon einmal eine eigene Sektion widmete (zu den Ergebnissen: Kammler \& Knapp, 2002), stand das Lüneburger Symposion 2004 ganz unter dem Tagungsthema "Deutschunterricht - empirisch».

8 Neuere Sprachbuchkonzeptionen waren beispielsweise zu finden in den Sprachbüchern «Schweizer Sprachbuch», «Welt der Wörter» oder in «Treffpunkt Sprache».

9 «In allen neueren Sprachbüchern wird die «Förderung der Kommunikationsfähigkeit, Verständigungsfähigkeit» als allgemeinstes Ziel des Sprachunterrichts erklärt.» (Sieber 1990, S. 7, Anm. 3).

10 Das Forum Deutschdidaktik als Arbeitsgruppe der Schweizerischen Gesellschaft für Lehrerinnen- und Lehrerbildung (SGL) engagiert sich für Austausch, Information und Koordination sowie Weiterbildung im Bereich der Deutschdidaktik, u.a. mit regelmässigen Treffen, Jahrestagungen und Informationen. Aktuelle Geschäftsstelle: PH Aargau, Prof. Dr. Thomas Lindauer: thomas.lindauer@fh-aargau.ch.

11 So beteiligten sich Schweizer KollegInnen an der Redaktion wichtiger fachdidaktischer Zeitschriften (Praxis Deutsch, Diskussion Deutsch, Der Deutschunterricht).

12 Was zunächst nur in kleinen Kreisen bekannt wurde, konnte so zu einer breiteren Öffentlichkeit finden. Dies war in den Folgejahren z. B. der Fall bei Konzepten des Lese-/Schreibunterrichts (Reichen, 1982) oder beim Konzept des dialogischen Lernens (Ruf \& Gallin, 1998).

13 Die seit 1996 erscheinende Zeitschrift des Symposion Deutschdidaktik Didaktik Deutsch hat sich - hervorgegangen aus einem früheren Mitgliederbrief («Postille») - zu einem 
wichtigen wissenschaftlichen Organ des Deutschdidaktik-internen Diskurses entwickelt: www.didaktik-deutsch.de. (5.11.2005).

14 Die Ergebnisse der ersten Projektphase («Literalität im medialen Umfeld») sind greifbar in Bertschi-Kaufmann, 2003, das zweite, umfassendere Projekt («Lernen im Kontext neuer Medien») hat die zusammenfassenden Ergebnisse zugänglich gemacht in Bertschi-Kaufmann, Kassis und Sieber (2004).

15 Um aus der Vielfalt nur einige zu nennen: Baurmann (2002); Fritzsche (1989); Liebnau (1995); Schader (2004); Spinner (2001); Themennummern von «Praxis Deutsch»: 161/2000 (Texte); 168/2001 (Schreibaufgaben); 182/2003 (Beschreiben und Beschreibungen).

16 Hilfreiche Hinweise zum Überarbeiten sind z.B. zusammengestellt in: Baurmann (2002); Baurmann und Ludwig (1996); Fix (2000); Portmann-Tselikas ( 2005); Themennummer von «Praxis Deutsch»: 179/2003 (Verständlich formulieren).

17 Die Diskussion wurde weitergeführt z.B. in Nussbaumer (1991, Kap. 8.2); Sieber (1994a, S. 318-332); Sieber (1998a, insbesondere Kap. 6) - und aktuell auf die Schule bezogen z.B. in: Bildungsdirektion des Kantons Zürich und Pädagogische Hochschule Zürich (2003, S. 10f.).

\section{Literaturverzeichnis}

Augst, G. \& Faigel, P. (1986). Von der Reihung zur Gestaltung. Untersuchungen zur Ontogenese der schriftsprachlichen Fähigkeiten von 13-23 Jahren (Theorie und Vermittlung der Sprache, 5). Frankfurt, Bern, New York: Lang.

Bachmann, T. (2002). Kohäsion und Kohärenz: Indikatoren für Schreibentwicklung. Zum Aufbau kohärenzstiftender Strukturen in instruktiven Texten von Kindern und Jugendlichen. Innsbruck: Studienverlag.

Bachmann, T., Bertschi-Kaufmann, A., Kassis, W., Schneider, H. \& Sieber, P. (2004). Resultate und Konsequenzen. In A. Bertschi-Kaufmann, W. Kassis, \& P. Sieber (Hrsg.), Mediennutzung und Schriftlernen. Analysen und Ergebnisse zur literalen und medialen Sozialisation. Unter Mitarbeit von T. Bachmann, H. J. Schneider und C. Tresch (S. 239-248). Weinheim und München: Juventa Verlag.

Bachmann, T. \& Sieber, P. (2004). Wechselwirkungen zwischen literaler Praxis und Schreibkompetenz. In A. Bertschi-Kaufmann, W. Kassis \& P. Sieber (Hrsg.), Mediennutzung und Schriftlernen. Analysen und Ergebnisse zur literalen und medialen Sozialisation. Unter Mitarbeit von T. Bachmann, H. J. Schneider und C. Tresch (S. 199-218). Weinheim und München: Juventa Verlag.

Baer, M., Fuchs, M., Reber-Wyss, M., Jurt, U. \& Nussbaum, T. (1995). Das «Orchester-Modell» der Textproduktion. In J. Baurmann, \& R. Weingarten (Hrsg.), Schreiben. Prozesse, Prozeduren und Produkte (S. 173-200). Opladen: Westdeutscher Verlag.

Barton, D. (1994). Literacy: an introduction to the ecology of written language. Oxford: Blackwell.

Baumgärtner, K., Fritz, G., Herrlitz, W., Hundsnurscher, F., Kastovsky, D., König, E. et al. (1973). Funk-Kolleg Sprache. Eine Einführung in die moderne Linguistik. (2 Bände, Fischer TB 6111, 6112). Frankfurt: Fischer.

Baurmann, J. (2002). Schreiben - Überarbeiten - Beurteilen. Ein Arbeitsbuch zur Schreibdidaktik. Seelze-Velber: Kallmeyersche Verlagsbuchhandlung.

Baurmann, J. (2003). Schulisches Schreiben im Schnittpunkt von Schreibdidaktik und Schreibforschung. In M. Kämper-van den Boogaart (Hrsg.), Deutsch-Didaktik: Leitfaden für die Sekundarstufe I und II (S. 249-262). Berlin: Cornelsen.

Baurmann, J. \& Ludwig, O. (1996). Schreiben: Texte und Formulierungen überarbeiten. Praxis Deutsch, 23, (137), 13-21. 
Baurmann, J. \& Weingarten, R. (Hrsg.). (1995). Schreiben. Prozesse, Prozeduren und Produkte. Opladen: Westdeutscher Verlag.

Becker-Mrotzek, M. (1997a). Schreibentwicklung und Textproduktion. Der Erwerb der Schreibfertigkeit am Beispiel der Bedienungsanleitung. Opladen: Westdeutscher Verlag.

Becker-Mrotzek, M. (1997b). Zum Verhältnis von Sprachwissenschaft und Sprachdidaktik. Didaktik Deutsch, 3, 16-32.

Beiträge zum Deutschunterricht I (1984). Lesen. Zürich: SLZ.

Beiträge zum Deutschunterricht II (1985). Texte schreiben. Zürich: SLZ.

Bereiter, C. (1980). Development in writing. In L. W. Gregg, \& E. R. Steinberg (Hrsg.), Cognitive Processes in Writing (p. 73-93). Hillsdale, N.J.: Lawrence Erlbaum Associates.

Bereiter, C. \& Scardamalia, M. (1987). The psychology of written composition. Hillsdale, N.J.: Lawrence Erlbaum Associates.

Bertschi-Kaufmann, A. (22003/2000). Lesen und Schreiben in einer Medienumgebung: die literalen Aktivitäten von Primarschulkindern. Aarau: Zentrum Lesen (1. Aufl.: Bildung Sauerländer).

Bertschi-Kaufmann, A., Kassis, W. \& Sieber, P. (Hrsg.). (2004). Mediennutzung und Schriftlernen: Analysen und Ergebnisse zur literalen und medialen Sozialisation. Unter Mitarbeit von T. Bachmann, H. J. Schneider und C. Tresch (Lesesozialisation und Medien). Weinheim und München: Juventa.

Bildungsdirektion des Kantons Zürich \& Pädagogische Hochschule Zürich (Hrsg.). (2003). Hochdeutsch als Unterrichtssprache: Befunde und Perspektiven. Redaktion: T. Bachmann, B. Good. Zürich: Bildungsdirektion.

Boettcher, W., Firges, J., Sitta, H. \& Tymister, H.-J. (51978/1973): Schulaufsätze - Texte für Leser. Düsseldorf: Schwann.

Bredel, U., Günther, H., Klotz, P., Ossner, J. \& Siebert-Ott, G. (Hrsg.). (2003). Didaktik der deutschen Sprache. Ein Handbuch (2 Teilbände, UTB 8235, 8236). Paderborn et al.: Ferdinand Schöningh.

Bremerich-Vos, A (1993). Grammatikunterricht - ein Plädoyer für das Backen kleinerer Brötchen. In A. Bremerich-Vos (Hrsg.), Handlunsgfeld Deutschunterricht im Kontext (S. 102129). Frankfurt: Diesterweg.

Burger, H. \& Häcki Buhofer, A. (Hrsg.). (1994). Spracherwerb im Spannungsfeld von Dialekt und Hochsprache (Zürcher Germanistische Studien, Band 38). Bern et al.: Lang.

Chandler, D. (1992). The Experience of Writing: A Media Theory Approach. PhD dissertation. Aberystwyth: University of Wales.

Cummins, J. (1980). The cross-lingual dimensions of language proficiency: Implications for bilingual education and the optimal age issue. In TESOL Quarterly, 14 (2), 175-187.

Cummins, J. (1991). Conversational and academic language proficiency in bilingual context. AILA Review, 8, 75-89.

Cummins, J. (2004). Language, Power and Pedagogy: Bilingual Children in the Crossfire (3rd ed.). Clevedon: Multilingual Matters.

Dehn, M. (1999). Texte und Kontexte: Schreiben als kulturelle Tätigkeit in der Grundschule. Berlin, Düsseldorf: Volk \& Wissen, Kamp.

Dürscheid, C. (2003). Medienkommunikation im Kontinuum von Mündlichkeit und Schriftlichkeit. Theoretische und empirische Probleme. Zeitschrift für Angewandte Linguistik, Heft 38, 37-56.

EDK (Eidgenössische Erziehungsdirektorenkonferenz) (Hrsg.). (2003). Aktionsplan «PISA 2000" - Folgemassnahmen. Beschluss der Plenarversammlung vom 12. Juni 2003. Bern: EDK.

EDK (2004). HarmoS - Zielsetzungen und Konzeption des Projekts, Juni 2004. Bern: EDK.

Eichler, W. \& Henze, W. (1990). Sprachwissenschaft und Sprachdidaktik. In G.Lange, K. Neumann. \& W. Zie-senis (Hrsg.), Taschenbuch des Deutschunterrichts. Grundfragen und 
Praxis der Sprach-und Literaturdidaktik. Band 1: Grundlagen - Sprachdidaktik-Mediendidaktik (4. Auflage, 1. korr. Nach-druck S. 153-198). Hohengehren: Schneider.

Europarat - Rat für Kulturelle Zusammenarbeit (Hrsg.). (2001). Gemeinsamer europäischer Referenzrah-men für Sprachen: lernen, lehren, beurteilen. Berlin et al.: Langenscheidt.

Feilke, H. (2002). Die Entwicklung literaler Textkompetenz. Ein Forschungsbericht (SPASS Siegener Papiere zur Aneignung sprachlicher Strukturformen). Siegen: Schriftenreihe der Universität-GH-Siegen, 10/2002.

Feilke, H. (2003). Entwicklung schriftlich-konzeptualer Fähigkeiten. In U. Bredel, H. Günther, P. Klotz, J. Ossner \& G. Siebert-Ott (Hrsg.), Didaktik der deutschen Sprache. Ein Handbuch. (2 Teilbände, S. 178-192). Paderborn et al: Ferdinand Schöningh.

Feilke, H. \& Portmann, P. R. (Hrsg.). (1996). Schreiben im Umbruch. Beiträge der linguistischen Schreibforschung zur Praxis und Reflexion schulischen Schreibens (Deutsch im Gespräch). Stuttgart et al.: Klett.

Fingerhut, K. (1995). Das Verhältnis von Fachwissenschaft und Fachdidaktik in der Zeit von 1964 bis 1994. In L. Jäger (Hrsg.), Germanistik: Disziplinäre Identität und kulturelle Leistung (S. 87-104). Weinheim: Beltz.

Fix, M. (2000). Textrevisionen in der Schule. Prozessorientierte Schreibdidaktik zwischen Instruktion und Selbststeuerung. Empirische Untersuchungen in achten Klassen. Hohengehren: Schneider.

Flower, L. S. \& Hayes, J. R. (1980). The Dynamics of Composing: Making Plans and Juggling Constraints. In L. W. Gregg \& E.R. Steinberg. (Hrsg.), Cognitive Processes in Writing (p. 31-50). Hillsdale (N.J.). Lawrence Erlbaum Associates.

Fritzsche, J. (1989). Schreibwerkstatt. Geschichten und Gedichte: Schreibaufgaben, -übungen, -spiele. Stuttgart: Klett.

Glinz, H. (2003). Geschichte der Sprachdidaktik. In U. Bredel, H. Günther, P. Klotz, J. Ossner \& G. Siebert-Ott (Hrsg.), Didaktik der deutschen Sprache. Ein Handbuch (S. 17-29). Paderborn et. al.: Ferdinand Schöningh.

Häcki Buhofer, A. (1985). Schriftlichkeit im Alltag. Theoretische und empirische Aspekte - am Beispiel eines Schweizer Industriebetriebs. Bern: Lang.

Hayes, J. R. \& Flower, L. S. (1980). Identifying the organization of writing processes. In L. W. Gregg \& E.R. Steinberg (Hrsg.), Cognitive Processes in Writing (p. 3-30). Hillsdale, N.J.: Lawrence Erlbaum Associates.

Hurrelmann, B. (1998). Deutschdidaktik - Kein Ort, nirgends? Didaktik Deutsch, Sonderheft, 13-38.

Kammler, C. \& Knapp, W. (Hrsg.). (2002). Empirische Unterrichtsforschung und Deutschdidaktik (Diskussionsforum Deutsch, Bd. 5). Baltmannsweiler: Schneider-Verlag Hohengehren.

Kämper-van den Boogaart, M. (2003). Fachdidaktik und Wissenschaft. In M. Kämper-van den Boogaart, (Hrsg.), Deutschdidaktik: Leitfaden für die Sekundarstufe I und II (S. 75-94). Berlin: Cornelsen Verlag Scriptor.

Koch, P. \& Oesterreicher, W. (1985). Sprache der Nähe - Sprache der Distanz. In Romanisches Jahrbuch, 36, 15-43.

Koch, P. \& Oesterreicher, W. (1994). Schriftlichkeit und Sprache. In H. Günther \& O. Ludwig (Hrsg.), Schrift und Schriftlichkeit. Writing and Its Use. Ein interdisziplinäres Handbuch internationaler Forschung. 1. Halbband. (Handbücher zur Sprach- und Kommunikationswissenschaft [HSK]10.1 S. 587-604). Berlin, New York: de Gruyter.

Köhler, G. \& Reuter, E. (Hrsg.). (1973). Was sollen Schüler lernen? Die Kontroverse um die hessischen Rahmenrichtlinien für die Unterrichtsfächer Deutsch und Gesellschaftslehre (Fischer TB 1460). Frankfurt: Fischer.

Liebnau, U. (1995). EigenSinn. Kreatives Schreiben - Anregungen und Methoden. Frankfurt: Diesterweg. 
Linke, A. \& Oomen-Welke, I. (1995). Herkunft, Geschlecht und Deutschunterricht: oben - unten / von hier - von anderswo / männlich - weiblich. Freiburg/Br.: Fillibach.

Lüdi, G., Boillat, J.-M., Bosshard, H.-U. \& Oertle Bürki, C. (1998). Welche Sprachen sollen die Schülerinnen und Schüler der Schweiz während der obligatorischen Schulzeit lernen? Bericht einer von der Kommission für Allgemeine Bildung eingesetzten Expertengruppe "Gesamtsprachenkonzept» an die Schweizerische Konferenz der Kantonalen Erziehungsdirektoren. Bern: EDK.

Ludwig, O. (1995). Integriertes und nicht-integriertes Schreiben. Zu einer Theorie des Schreibens: eine Skizze. In J. Baurmann \& R. Weingarten (Hrsg.), Schreiben. Prozesse, Prozeduren und Produkte (S. 273-287). Opladen: Westdeutscher Verlag.

Ludwig, O. (1996). Vom diktierenden zum schreibenden Autor. In H. Feilke \& P. R. Portmann (Hrsg.), Schreiben im Umbruch. Beiträge der linguistischen Schreibforschung zur Praxis und Reflexion schulischen Schreibens (Deutsch im Gespräch S. 16-28). Stuttgart et al.: Klett.

Ludwig, O. (2001). Es begann mit dem Sputnik-Schock...Die neuere deutsche Schreibforschung. Praxis Deutsch, 28, (170), 58-62.

Ludwig, O. (2003a). Konzeptionen des Schreibens. In Der Deutschunterricht, 3, 4-13.

Ludwig, O. (2003b). Entwicklung schulischer Schreibdidaktik in Deutschland und ihr Bezug zum akademischen Schreiben. In K. Ehlich \& A. Steets (Hrsg.), Wissenschaftlich schreiben - lehren und lernen. (S. 235-250). Berlin, New York: de Gruyter.

Molitor-Lübbert, S. (1996). Schreiben als mentaler und psychischer Prozess. In H. Günther \& O. Ludwig (Hrsg.), Schrift und Schriftlichkeit. Writing and Its Use. Ein interdisziplinäres Handbuch internationaler Forschung (2. Halbband S. 1005-1027). (Handbücher zur Sprach- und Kommunikationswissenschaft [HSK] Band 10.2). Berlin, New York: de Gruyter.

Müller-Michaels, H. (1994). Konzepte des Deutschunterrichts nach 1968. In J. S. Hohmann (Hrsg.), Deutschunterricht zwischen Reform und Modernismus. Blicke auf die Zeit 1968 bis heute (S. 27-43). Frankfurt: Lang.

Nussbaumer, M. (1991). Was Texte sind und wie sie sein sollen. Ansätze zu einer sprachwissenschaftlichen Begründung eines Kriterienrasters zur Beurteilung schriftlicher Schülertexte (Reihe Germanistische Linguistik (RGL) 119. Tübingen: Niemeyer.

Nussbaumer, M. \& Sieber, P. (1995). Über Textqualitäten reden lernen - z.B. anhand des "Zürcher Textanalyserasters". Diskussion Deutsch, 141, 36-52.

Oelkers, J. (2002): "Und wo, bitte, bleibt Humboldt?". In Die Zeit Nr. 27, 27. Juni 2002. S. 36.

Oelkers, J. (2002): Gut schreiben können ist mehr als eine SMS verfassen. NZZ am Sonntag 1. September 2002. S. 19.

Ong, W. J. (1982/1987). Oralität und Literalität. Die Technologisierung des Wortes. Opladen: Westdeutscher Verlag.

Ortner, H. (2000). Schreiben und Denken (Reihe Germanistische Linguistik (RGL) 214). Tübingen: Niemeyer.

Portmann, P. R. (1991). Schreiben und Lernen. Grundlagen der fremdsprachlichen Schreibdidaktik (Reihe Germanistische Linguistik (RGL) 122). Tübingen: Niemeyer.

Portmann-Tselikas, P. R. (2002). Textkompetenz und unterrichtlicher Spracherwerb. In P. R. Portmann-Tselikas, \& S. Schmölzer-Eibinger (Hrsg.), Textkompetenz: Neue Perspektiven für das Lernen und Lehren (S. 13-43). Innsbruck et al.: StudienVerlag.

Portmann-Tselikas, P. R. (2005). Schreiben und Überarbeiten von Texten. In U. Abraham, C. Kupfer-Schreiner \& K. Maiwald (Hrsg.), Schreibförderung und Schreiberziehung: Eine Einführung für Schule und Hochschule (S. 174-186). Donauwörth: Auer Verlag.

Reichen, J. (1982). Lesen durch Schreiben. Wie Kinder selbstgesteuert Lesen lernen. Lesedidaktische, lernpsychologische und schulpädagogische Grundlagen eines vom Schüler selbstgesteuerten Schriftspracherwerbs. Zürich: SABE. 
Ruf, U. (Hrsg.).(41992/1987). Rechtschreibunterricht. Zürich: Verlag des Schweizerischen Lehrervereins.

Ruf, U. \& Gallin, P. (1998). Dialogisches Lernen in Sprache und Mathematik. Bd. I: Austausch unter Ungleichen: Grundzüge einer interaktiven und fächerübergreifenden Didaktik. Bd. II: Spuren legen - Spuren lesen: Unterricht mit Kernideen und Reisetagebüchern. Seelze-Velber: Kallmeyersche Verlagsbuchhandlung..

Schader, B. (2004). Sprachenvielfalt als Chance: Das Handbuch. Hintergründe und 101 praktische Vor-schläge für den Unterricht in mehrsprachigen Klassen. Troisdorf, Zürich: Bildungsverlag EINS, Orell Füssli.

Sieber, P. (1990). Perspektiven einer Deutschdidaktik für die deutsche Schweiz (Reihe Sprachlandschaft, 8). Aarau, Frankfurt, Salzburg: Sauerländer.

Sieber, P. (Hrsg.).(1994a). Sprachfähigkeiten - Besser als ihr Ruf und nötiger denn je! Ergebnisse und Folgerungen aus einem Forschungsprojekt (Reihe Sprachlandschaft, 12). Aarau, Frankfurt, Salzburg: Sauerländer.

Sieber, P. (1994b). Konzepte des Lernens - Bilder von Lernenden. In Schweizer Schule, 7-8, 3-15.

Sieber, P. (1998a). Parlando in Texten. Zur Veränderung kommunikativer Grundmuster in der Schriftlichkeit (Reihe Germanistische Linguistik (RGL) 191). Tübingen: Niemeyer.

Sieber, P. (1998b). Sprachwissenschaft und Sprachdidaktik - zum Verhältnis zweier ungleicher Schwestern. Beiträge zur Lehrerbildung (BzL) 16 (3), 353-366.

Sieber, P. (2000). Schreiben im Spannungsfeld von Oralität und Literalität. In H.Witte, C. Garbe, K. Holle, J. Stückrath \& H. Willenberg (Hrsg.), Deutschunterricht zwischen Kompetenzerwerb und Persönlichkeitsbildung (S. 114-133). (Diskussionsforum Deutsch, Band 2). Hohengehren: Schneider.

Sieber, P. (2003a). Art. 15. Modelle des Schreibprozesses. In U. Bredel, H. Günther, P. Klotz, J. Ossner \& G. Siebert-Ott (Hrsg.), Didaktik der deutschen Sprache - ein Handbuch (S. 208-223). (Band 1, UTB 8235). Paderborn et al.: Ferdinand Schöningh.

Sieber, P. (2003b). Hatte die germanistische Sprachwissenschaft Einfluss auf die Auffassungen von Sprache im Deutschunterricht? In H. Henne, H. Sitta \& H. E. Wiegand (Hrsg.), Germanistische Linguistik: Konturen eines Faches (S. 117-136). (Reihe Germanistische Linguistik (RGL) 240). Tübingen: Niemeyer.

Sieber, P. \& Sitta, H. (1986). Mundart und Standardsprache als Problem der Schule (Reihe Sprachlandschaft, 3). Aarau, Frankfurt, Salzburg: Sauerländer.

Sieber, P. \& Sitta, H. (1994). Symposion Deutschdidaktik in Zürich. Beiträge zur Lehrerbildung (BzL) 12 (2), 141-145.

Sitta, H. (1979). Spracherwerbstheoretische Aspekte des Verhältnisses von Mundart und Hochsprache in der Schule. In H. Löffler, K. Pestalozzi \& M. Stern (Hrsg.), Standard und Dialekt. Studien zur gesprochenen und geschriebenen Gegenwartssprache. Festschrift für Heinz Rupp zum 60. Geburtstag (S. 165-175). Bern, München: Francke.

Spinner, K. (1994). Neue und alte Bilder von Lernenden. Deutschdidaktik im Zeichen der kognitiven Wende. Beiträge zur Lehrerbildung (BzL) 12 (2), 146-158.

Spinner, K. (2001). Kreativer Deutschunterricht. Identität - Imagination - Kognition. SeelzeVelber: Kallmeyersche Verlagsbuchhandlung.

Spitta, G. (1992). Schreibkonferenzen in Klasse 3 und 4. Ein Weg vom spontanen Schreiben zum bewussten Verfassen von Texten. Berlin: Cornelsen, Scriptor.

Stern, O. (31994/1988). Wie lernen Kinder in der Deutschschweiz Hochdeutsch? In P. Sieber $\&$ H. Sitta (Hrsg.), Mundart und Hochdeutsch im Unterricht. Orientierungshilfen für Lehrer (S. 23-36). (Studienbücher Sprachlandschaft, 1). Aarau, Frankfurt, Salzburg: Sauerländer.

Werlen, E. (1993). Dialekt als Norm. Hochdeutsch als Abweichung. Zur Situation von Dialekt und Schule in der deutschsprachigen Schweiz. In P. Klotz \& P. Sieber (Hrsg.), Vielerlei Deutsch. Umgang mit Sprachvarietäten in der Schule (Deutsch im Gespräch) (S. 94-109). Stuttgart: Klett. 
Werlen, I. (2004). Zur Sprachsituation der Schweiz mit besonderer Berücksichtigung der Diglossie in der Deutschschweiz. In Bulletin VALS/ASLA, 79, 1-30.

Schlagworte: didattica della scrittura, ricerca della scrittura, scrittura processuale, competenza testuale

\section{Didactique de l'écriture: du produit vers le processus, et jusqu'à la compétence textuelle}

\section{Résumé}

Comment la discussion sur la didactique de l'écriture dans le monde germanophone, au cours des dix dernières années s'est-elle développée? Les lignes essentielles d'articulation sont ici tracées, avec une attention particulière aux discussions et contributions en provenance de la Suisse alémanique. Grâce à la coopération positive entre recherche sur l'écriture et didactique, des bases empiriques pour la didactique de l'écriture ont pu être construites. L'attention au processus d'écriture a, sur ce point, pris dans les dix dernières années une importance centrale. Une didactique de l'écriture «orientée processus» a engendré une multiplicité d'innovations pratiques facilitant le succès de cet enseignement. Tout récemment, à la lumière du débat public sur les performances scolaires, les effets visés ont mieux été pris en compte et la nécessité d'une large promotion des compétences littéraires a mis la construction de compétences textuelles au centre des recherches.

Mots clés: didactique de l'écriture, compétences textuelles, processus d'écriture, recherche sur l'écriture, développement de la didactique disciplinaire

\section{Didattica della scrittura - dal prodotto al processo fino alla competenza testuale}

\section{Riassunto}

Come si è sviluppata negli ultimi decenni la discussione attorno alla didattica della scrittura nell'area tedescofona? L'articolo ricostruisce importanti linee di tendenza concedendo uno sguardo particolare alla discussione e ai contributi nella Svizzera tedesca. Si constata che una cooperazione produttiva tra ricerca e didattica della scrittura hanno reso possibile una base empirica per la didattica della scrittura stessa. L'attribuzione di un ruolo centrale al processo di scrittura verificatosi negli ultimi anni ha altresì dato luogo ad una "didattica della scrittura processuale" e a numerosi stimoli per un insegnamento della scrittura efficace. Recentemente, anche nell'ottica della discussione pubblica delle prestazioni della scuola, gli effetti di questo insegnamento sono oggetto di particolare attenzione 
e di dibattito: così con l'accresciuta promozione scolastica delle competenze letterali anche la competenza testuale specifica sta diventando un oggetto di ricerca privilegiato.

Parole chiave: Didaktik des Schreibens ; Kooperation von Schreibforschung und Schreibdidaktik ; «prozessorientierte Schreibdidaktik»; breite Literalitätsförderung; Aufbau von Textkompetenz

\section{Writing Didactics - From Product to Process to Text Competence}

\section{Summary}

How did the discussion about the teaching and learning of writing evolve in the German speaking area during the last decades? This paper reconstructs important lines of development, paying special attention to discussions and contributions from German-speaking Switzerland. An empirical foundation for teaching and learning of writing has been built up through a productive cooperation between research on writing, and writing didactics. Within this cooperation, the view on the process of writing has become a central issue. Based on this foundation, process-oriented writing didactics have brought forth a number of suggestions for successful writing didactics. Recently - in part due to a higher public interest in school achievement -, more attention has been paid to the effects of this teaching, leading to more frequent discussions. As the promotion of literacy has become a societal necessity, the issue of text competence is an increasingly important topic of research.

Key words: Writing didactics; cooperation of research of the writing activity and writing didactics ; "process-oriented writing didactics»; wide promotion of literality 